(Presented at Conference on High Angular Momentum Properties of Nuclei, Oak Ridge, Tennessee, November 2-4, 1982)

$$
\text { CONF- } \times 21143-13
$$

CONF-821143--13

LIFETIMES OF BIGH-SEIN STATES OF 160,161YD

DE\&4 003277

M.P. FEhELI, C. BAKTASH, M.h. GUIDRY, J J.S. RATTULA, 2

N.R. JOENSON, I.Y. LEE, F.K. MCGOWAN, H. ONER, I

S. C. PANCHOLI; ${ }^{3}$ L.L. RIEDINGER ${ }^{1}$ ANO J.C. WELIS ${ }^{4}$

Ozk Ridge National Laboratory, Oak Ridge, Tennessee, U.S.A.

Ebstract Recoll-distance measurements of lifetides of high-spin states of $160.161 \mathrm{Yb}$ are described. While the resuits for the ground band of $160 \mathrm{Yb}$ are not inconsistent with centrifugal stretching, the addition of one or more quasineutrons seems to cause a reduction in collectlvisy which depends on frequencs but not, for the ceses examined, on the type of quasineutron.

\title{
INTRODUCTION
}

The use of the recoll-distance method with fusion-evaporation reactions permits the measurement of lifetimes of high-spin states. From these data, one may examine the variation of nuclear deformation with angular momentum. The nucleus $160 \mathrm{gb}$ is known to have a strong backbend at about spin 12 and a number of weli aligned sidebands, ${ }^{5}$ giving the possibility of studying the deformation for different configurations of the quasineutrons.

\section{EXPERINENTAI METHOD}

Yxterbium isotopes were the by the reaction $116_{\mathrm{Cd}}\left(4 \mathrm{E}_{\mathrm{Ti}}, \mathrm{mn}\right)$ and its inverse ${ }^{45 \mathrm{Ti}}(216 \mathrm{Cd}, \mathrm{mm})$. In the former case, a 205-MeV ${ }^{4} 8_{\text {Ti }}$ beam from the $25-M V$ electrostatic accelerator at Oak Ridge was used, leading to a recoil velocity of $6.87 \mathrm{rm} / \mathrm{ps}$. The $116 \mathrm{Ca}$ beam was obtained by using the electrostatic accelerator as an injector for ORIC. The beam

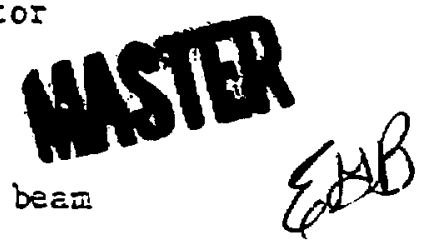

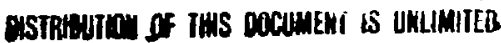




\section{DISCLAIMER}

This report was prepared as an account of work sponsored by an agency of the United States Government. Neither the United States Government nor any agency the reof, nor any of their employees, makes any warranty, express or implied, or assumes any legal liability or responsibility for the accuracy, completeness, or usefulness of any information, apparatus, product, or proces, disclosed, or represents that its use would not infringe privately owned rights. Refer$\mathrm{e}_{1,}$; herein to any specific commercial product, process, or service by trade name, trademark, manufacturer, or otherwise does not necessarily constitute or imply its endorsement, recommendation, or favoring by the United States Government or any agency thereof. The views and opinions of authors expressed herein do nol necessarily state or reflect those of the United States Government or any agency thereof.

\section{It has been reproduced irom iLLEIBLE availatule cepy to permit rom ihe hest possible availabilticy.}


energy, $495 \mathrm{MeV}$, was chosen to give the same center-of mass energy as in the titanium-induced reaction. This gave a recoll velocity of $19.3 \mathrm{um} / \mathrm{ps}$.

Our method differed in two respects from the standard recoil-distance technique. In order to provide some selection of reaction channel, we ${ }^{6}$ have constructed a recoildistance device which fits into the annular opening of a 25-cm $\times 25-\mathrm{cm}$ NaI detector, as illustrated in Fig. 1 . It is designed so that the rarget foil is located near the center of the NaI crystal which is operated as a total-energy sum spectrometer. The "stopper" to catch the recoiling nuclei consisted of a high qualitg nickel foil onto which about $40 \mathrm{mg} / \mathrm{cm}^{2}$ of lead was evaporated.

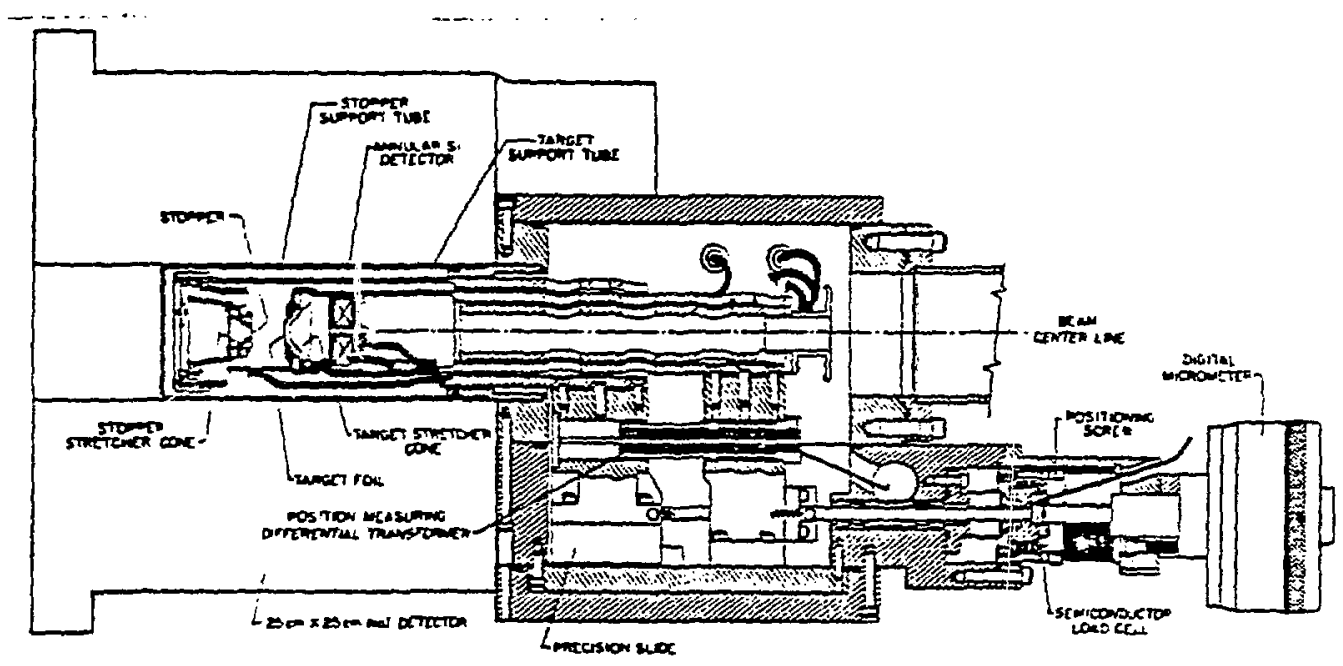

FIGURE 1. Schematic drawing of the Oak Ridge Recoil Distance Device. All of the principal components are labeled. The germanium detector for the system is not shown, but it fits in the annular opening of the NaI detector opposite the plunger mechanism. 


\section{IIFETINES OF HIGE-SPIN STATES OF ${ }^{160,161}$ YO}

The second modification to the usual recoil-distance techntque was adopted for the Inverse reaction. In order to avold the severe Doppler broadening of the "unshifted" peaks due to the high recoll velocity, we replaced the stopper foll with a gold foll of thickness $4.5 \mathrm{mg} / \mathrm{cm}^{2}$. This slowed the recoils to $13.5 \mathrm{~mm} / \mathrm{ps}$. The recoils and the beam were finally stopped by a $30 \mathrm{mg} / \mathrm{cm}^{2}$ lead foil positioned behind the gold foil such that only the first $2^{+}$state and ground state had appreciable population after the recoils had come to rest. Thus, at large target-retarder separations, the $2^{+}+0^{+}$transition appeared as three peaks - an unshifted, a less shifted, and a fully shifted. These were used to determine the recoil velocity.

Spectra were taken at eleven target-stopper separations with the ${ }^{48}$ TI beam and six target-retarder separations with the ${ }^{116} \mathrm{Cd}$ beam. Our primary interest in the inverse reaction ias to obtain data at small target-retarder separations in order to gain some information about the states near the second backbend at spin $28^{+}$. Unfortunately, target problems prevented this, but useful additional points on the decay curves of the yrast states below the $20^{+}$were obtained. Figure 2 shows limited energy regions in the gated $\mathrm{Ge}(\mathrm{Li})$ spectra for some of the target-retarder distances. Because of the complexity of the spectra, it was not possible to obtain the areas of both the shifted and unshifted peaks of all transitions. To normalize the spectra obtained at different target-stopper separations, we chose to use the intensity of the continuum above the discrete peaks. 
M. P. FEWELL ET AL.

FIGURE 2. Sections of the $Y$-ay spectra in the " $4 \mathrm{n}$ " gate recorded with flight ttmes between the target and retarding foll of (a) 137 ps, (b) $29 \mathrm{ps,} \mathrm{(c)} 9.0 \mathrm{ps}$ for the Cd-induced reaction. On'y wembers of the yrast sequence are labeled.

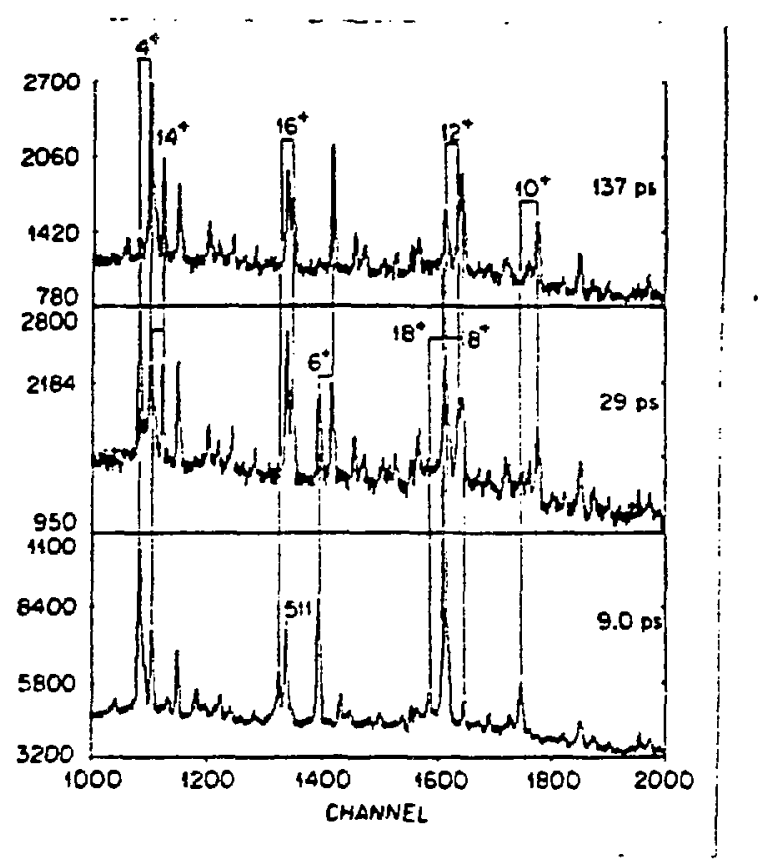

\section{RESULTS}

The major problem in extracting lifetimes from the decay curves is that of feeding. In a (EI, $x n$ ) reaction the observed levels are populated through transitions from other unobserved levels whose lifetimes are, in general, comparable to those being measured. We have attacked the problem with an extensively modified version of a computer program originally due to H. Emling of G.S.I., Darmstadt. This program applies all of the usual corrections ${ }^{7}$ to the data, but handles the problem of feeding by direct solution of the Bateman equations. That is, given an ansatz for the decay scheme, the program adjusts the lifetimes and initial populations of the levels to obtain the best fit to the decay curves. This ansatz is shown in Fig. 3. At each point where sidefeeding is shown in Fig. 3, a cascade of two levels was used to model the time distribution of the sidefeeding intensity. 
LIFETIMES OF HIGE-SPIN STATES OF $160,161_{\mathrm{Yb}}$

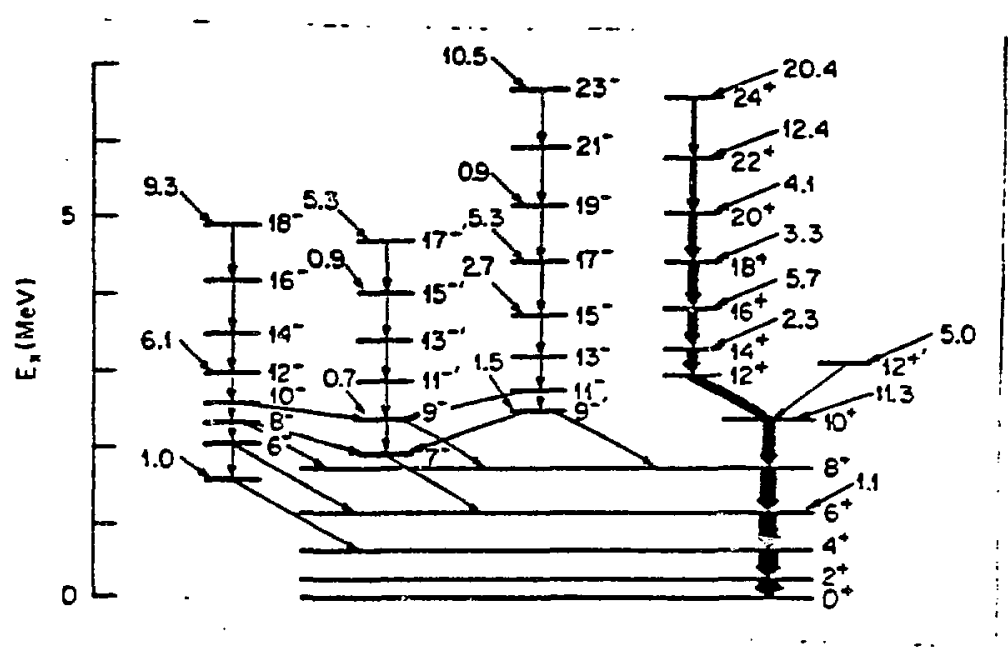

FIGURE 3. Sidefeeding intensities as a percentage of the $2^{+} \rightarrow 0^{+}$transition intensity. Decay curves have been obtalned for most of the transitions shown.

Before turning to the results of this analysis, let us consider some preliminary results of cranked HFB calculations carried out recently by Aberg, Bengtsson, Zhang and Chen. ${ }^{B}$ They find that the ground band of 160 yo starts out at $\varepsilon_{2}=0.18$ and $\gamma=1^{\circ}$ and reaches values of $\varepsilon_{2}=0.22$ and $\gamma^{2}=-5^{\circ}$ at $\operatorname{spin} 8^{+}$. The s-band, however, starts out at the $10^{+}$state with $\varepsilon_{2}=0.19, \gamma=4^{\circ}$ and by $18^{+}$has continued to maintain a fairly constant quadrupole deformation $\varepsilon_{2}$, while $\gamma$ has steadily increased to about $10^{\circ}$ $\left(\gamma=+60^{\circ}\right.$ corresponds to oblate shape, non-collective rotation).

In the following figures, we express our results as transition quadrupole moments $Q_{t}$, defined by

$$
B(E 2+)=\frac{5}{16 \pi}\left(I_{1} 200 \mid I_{f} 0\right)^{2} Q_{t}{ }^{2} \text {, }
$$

and compare these to the Intrinsic quadrupole moments $Q_{0}$ 
M. P. FEWELL ET AI.

computed from the value $\varepsilon_{2}$ and $\gamma$ corresponding to the intilal or decaying level according to 9

where

$$
Q_{0}=4 / 3 z e\left\langle I^{2}\right\rangle \frac{\cos \left(30^{\circ}+\gamma\right)}{\cos \left(30^{\circ}\right)} \cdot E_{2} \text {, }
$$

$$
\left\langle I^{2}\right\rangle=3 / 5\left(1.25 \mathrm{~A}^{1 / 3}\right)^{2} \mathrm{fm}^{2}
$$

Figure 4 shows the $Q_{t}$ values of those $g$-band members of 260

Yb for which we currently have values. The value for the $6^{+}$state is preliminary. The difficulty in obtaining Iifetimes for the higher g-band members arises from the complex feeding patterns from the sidebands into the $8^{+}$and $6^{+}$states (see FIg. 3). Although calculations ${ }^{8}$ show some evidence for "stretching" in the ground band, it is difficult to assess whether or not the limited experimental data substantiate or refute this.

FIGURE 4. A comparison of measured and theoretical quadrupole moments of $g$-bard members of ${ }^{260} \mathrm{Yb}$. The open circles are from the experimental data.

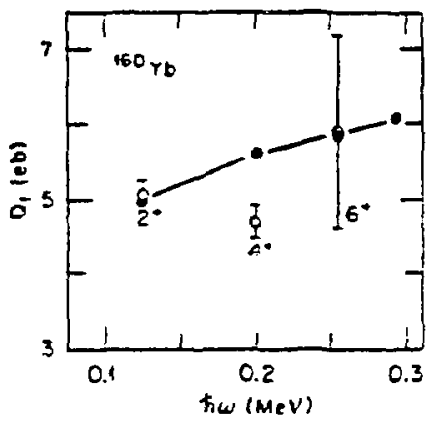

Figure 5 shows $Q_{t}$ values for various quasiparticle bands in 160,161 Yo. While the results in Fig. 4 are not inconsistent with centrifugal stretching, the results for 161 Yb in Fig. 5 show that the addition of one quasiparticle produces the opposite behavior. The two-quasiparticle bands of ${ }^{160} \mathrm{Yb}$ show a similar effect, but apparently with an increased rate of loss of collectivity with increasing rotational frequescy. This latter difference, which we failed 
to recognize in our abstract due to a miscalculation of the $Q_{t}$ value for the $17 / 2^{+}$state of ${ }^{161} \mathrm{Yb}$, is probably due to the $N=88$ core of ${ }^{160} \mathrm{~Tb}$ being softer and wore easily deformed than the $\mathrm{N}=90$ core of ${ }^{161} \mathrm{~Tb}$. The results for ${ }^{160} \mathrm{Yb}$ also suggest that two $1_{13 / 2}$ quasineutrons have approximately the same effect on the core as an $i 13 / 2^{\mathrm{h}} 9 / 2$ pair.

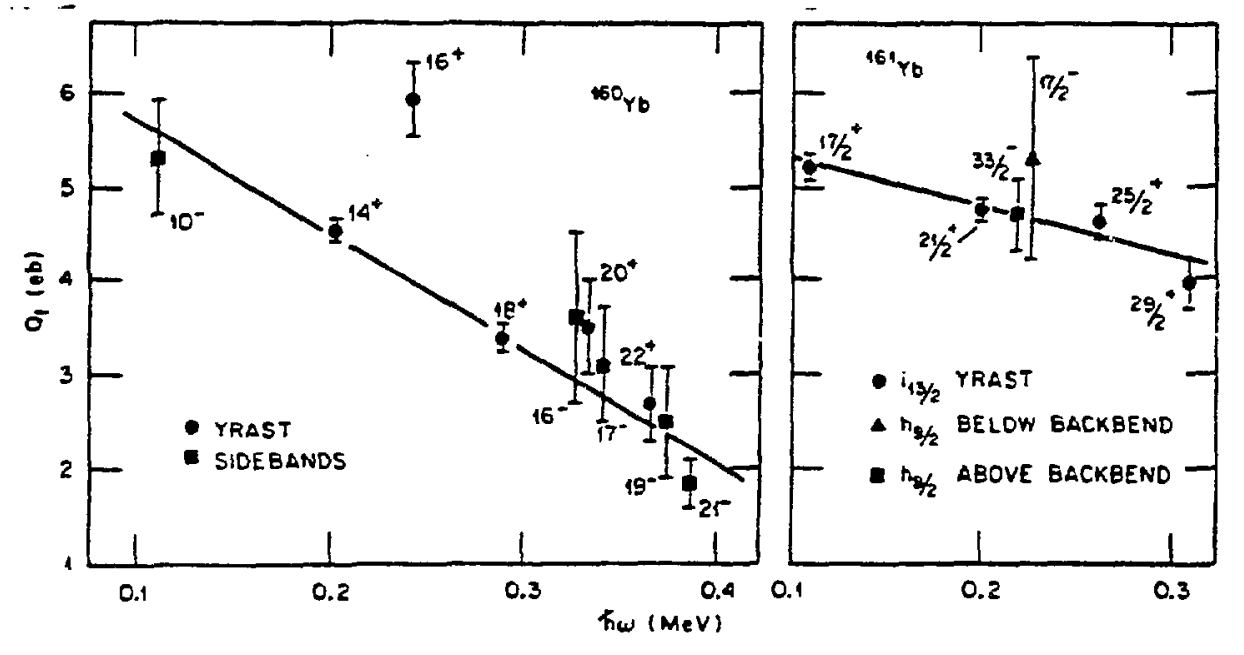

FIGURE 5. Quadrupole moments of some quasiparticleband members of 160,161 Yo versus rotational frequency.

Finally; Fig. 6 presents a comparison of the results for the s-band of ${ }^{160} \mathrm{Yb}$ and the theoretical calculations. 8 our result for the $16^{+}$yrast state is difficult to understand. It is unlikely that it is due to a mistake in allowing for feeding, firstiy because the inferred lifetime is shorter than expected, and secondly because only $14 \%$ of the feeding of the $16^{+}$level is due to unobserved sidefeeding, the remainder coming from the $18^{+}+16^{+}$transition (see Fig. 3). If the dropoff in $Q_{t}$ in Fig. 6 does indeed 
arise from increasing positive $r$-deformation, it appears that the theory falls quantitatively in predicting the large degree of triaxiality at the higher rotational frequencies.

FIGURE 6. A comparison of measured and theoretical quadrupole moments of s-band members of ${ }^{16}{ }^{\circ} \mathrm{Yb}$. The closed circles are the theoretical values.

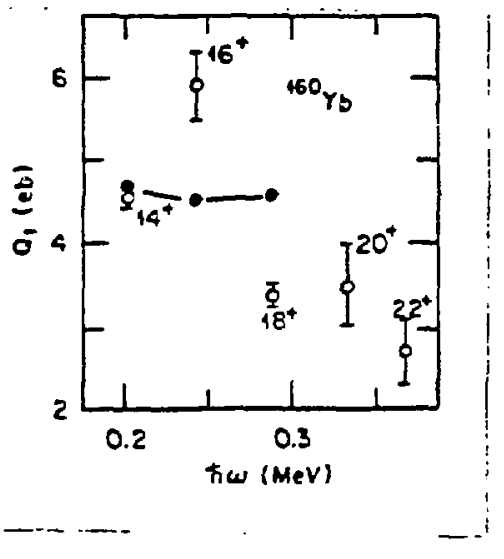

ACRNOWLEDGMENTS

This work was supported by the U.S. Department of Energy under Contract No. W-7405-eng-26 with Union Carbide Corporation.

\section{REFERENCES}

1. University of Tennessee, Knoxville, Tennessee.

2. University of Jyvaskyla, Finland.

3. University of Delhi, India, and Vanderbilt University, Nashville, Tennessee.

4. Tennessee Technological University, Cookeville, Tennessee.

5. I.I. Riedinger et al., Phys. Rev. Lett. 44, 568 (1980) and Proc. Nordic Conf. On Nucl. Phys., Fugls $\phi, 1982$, Phys. Scripta, in press.

6. N.R. Johnson, et al., ORNL Phys. Div. Ann. Prog. Rep., Novemiver 1982, ORNL-5787, P. 147 .

7. R.J. Sturm and M.W. Guidry, Nucl. Instr. Meth. 138, 345 (1976).

8. S. Áberg, R. Bengtsson, J.Y. Zhang and Y.S. Chen, private comunication.

9. P. Ring et al., Phys. Lett. 110B, 423 (1982). 